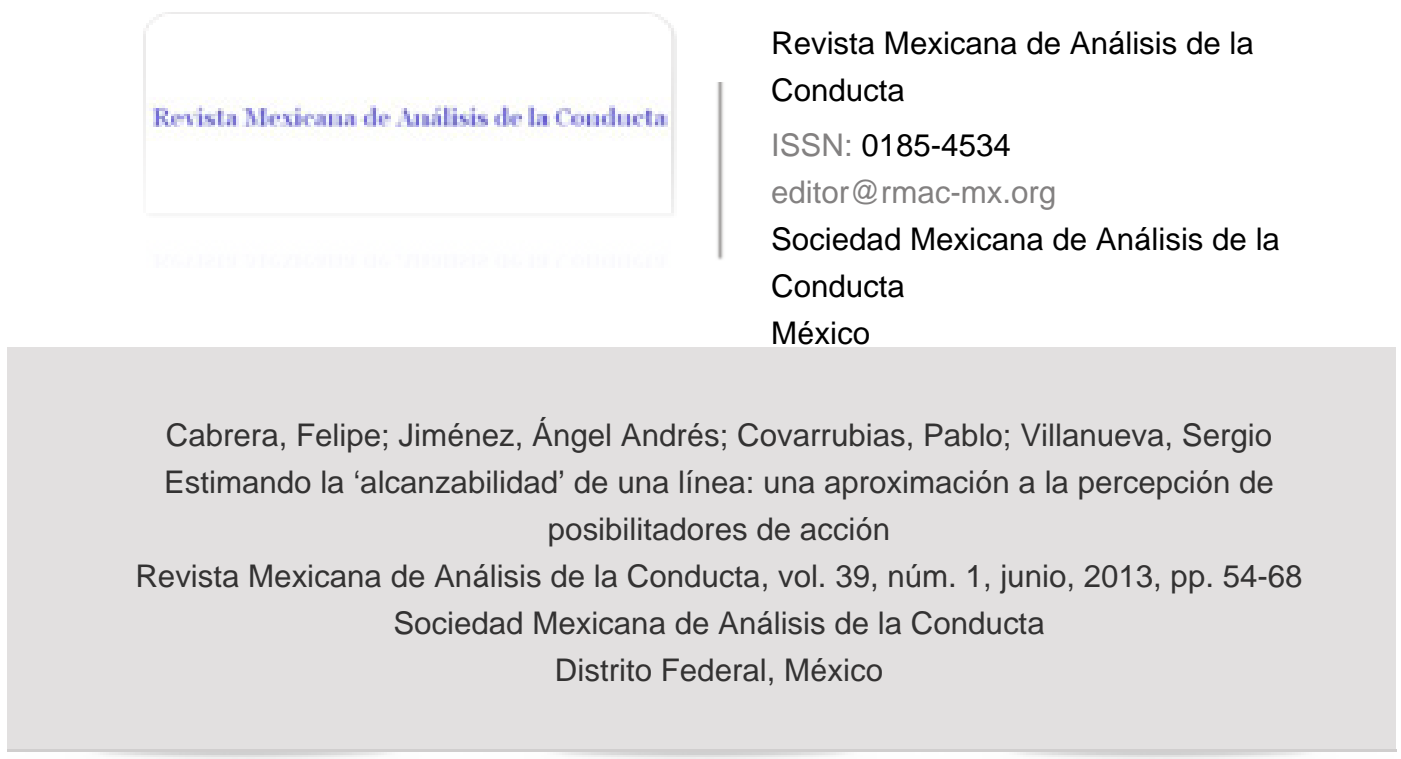

Disponible en: http://www.redalyc.org/articulo.oa?id=59335807005

- Cómo citar el artículo

- Número completo

- Más información del artículo

- Página de la revista en redalyc.org

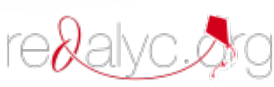

Sistema de Información Científica

Red de Revistas Científicas de América Latina, el Caribe, España y Portugal Proyecto académico sin fines de lucro, desarrollado bajo la iniciativa de acceso abierto 


\title{
ESTIMANDO LA 'ALCANZABILIDAD’ DE UNA LÍNEA: UNA APROXIMACIÓN A LA PERCEPCIÓN DE POSIBILITADORES DE ACCIÓN
}

\author{
ESTIMATING THE 'REACHABILITY' OF A LINE: AN AFFORDANCE \\ PERCEPTION APPROACH
}

\author{
FELIPE CABRERA, ÁNGEL ANDRÉS JIMÉNEZ, PABLO COVARRUBIAS \\ UNIVERSIDAD DE GUADALAJARA -CUCIÉNEGA. LABORATORIO \\ DE CONDUCTA Y COGNICIÓN COMPARADA \\ SERGIO VILLANUEVA \\ UNIVERSIDAD DEL VALLE DE MÉXICO-CAMPUS HISPANO
}

\begin{abstract}
Resumen
En dos experimentos se evaluó la precisión con la que participantes estimaron la 'alcanzabilidad' de una línea horizontal que se presentó a diferentes alturas. En el Experimento 1 los participantes basaron sus estimaciones en el alcance máximo vertical de su brazo, en el Experimento 2 las estimaciones se hicieron con respecto a una barra cuya longitud era igual al alcance máximo vertical de los participantes. Ambos experimentos consistieron en dos condiciones: una en la que los participantes hicieron sus estimaciones sentados sobre una silla de pequeña altura (soporte bajo) y otra en la que se sentaron sobre una silla de mayor altura (soporte alto). El análisis de los datos se hizo siguiendo la métrica intrínseca en el que la precisión en la estimación de los participantes se expresó como el cociente de la altura del estímulo sobre el alcance máximo vertical del brazo o de la extensión de la barra. Los resultados mostraron que la estimación de los participantes fue más precisa cuando se hizo con base en el propio brazo (Experimento 1) que con la barra (Experimento 2). Asimismo, en ambos experimentos los participantes mostraron menor certeza en sus estimaciones

\footnotetext{
El primer autor agradece a CONACyT financiamiento CB-2012-01-180443.

Agradecimientos especiales a Claudia E. Peralta por colaborar en desarrollo y registro en el Experimento

Enviar correspondencia a primer autor a: fcabrera@cencar.udg.mx

Laboratorio de Conducta y Cognición Comparada, Centro Universitario de la Ciénega. Av. Universidad
} 1, y a Priscila Carrillo por colaboración en desarrollo y registro del Experimento 2. 1115, CP 47820 Ocotlán, Jalisco, México.
\end{abstract}


a alturas cercanas al límite crítico absoluto. Los hallazgos se discuten en términos del significado conductual de los estímulos, la métrica intrínseca en la percepción de los posibilitadores de acción y la posible aportación de dichos hallazgos para el análisis experimental de la conducta.

Palabras clave: posibilitadores de acción, métrica intrínseca, soporte conductual, percepción, humanos

\begin{abstract}
Two experiments were conducted to assess the perception of 'reachability' of horizontal lines at different heights in humans. In Experiment 1, participants were asked whether the lines were reachable using their arms, and in Experiment 2 the estimation was made using a pole that was sized at the maximum extension of the participants' arms in vertical position. Two conditions were arranged for both experiments: participants estimated whether the lines were reachable while sitting in a small chair (Low Support Condition) or while sitting in a tall chair (High Support Condition). For data analysis we used a body-scaled or intrinsic metric that is expressed as the ratio of the height of stimuli with respect to the participants' maximum arm extension or respect to the pole's extension. This measurement evaluated the accuracy of the participants' estimation. Results showed that subjects estimated the reachability of the lines more accurately when using their arms (Experiment 1 ) than when using the pole (Experiment 2); nevertheless, participants had less confidence of their estimations at heights close to the absolute critical boundary. Findings are discussed in terms of the behavioral meaning of stimuli, the intrinsic metric in the affordance perception, and their contribution for the experimental analysis of behavior.

Keywords: affordances, intrinsic metric, behavioral support, perception, humans
\end{abstract}

En el análisis experimental de la conducta (AEC), los tópicos referentes a la percepción y la psicofísica están asociados a los procedimientos de control de estímulos (Blough, 1958; Staddon, 1983), de tal manera que la experiencia perceptual queda definida a partir del control discriminativo de los estímulos (Skinner, 1953, 1974). Este control discriminativo se establece en virtud de la contingencia de reforzamiento con el estímulo antecedente, es decir, de constituir la ocasión para obtener reforzamiento (Honig \& Urcuioli, 1981; Nevin, Davison, \& Commons, 1991; Terrace, 1966). No obstante, en el AEC escasamente se ha abordado el efecto conductual del estímulo antecedente por sí mismo, independientemente del reforzamiento (para un análisis crítico, ver Tonneau, 2012), dado que su énfasis principal es en la conducta guiada por sus consecuencias (Staddon, 1983).

Una manera en la que se ha hecho referencia a los estímulos antecedentes, en momentos previos al establecimiento de la contingencia de reforzamiento, es con el término 'proto-elementos' de la contingencia (Timberlake, 2004). Este término incluye todo elemento presente en la situación con el que el organismo entra en contacto, 
CABRERA ET AL.

tal como las luces, piso y paredes, sonidos, la palanca o tecla, comedero, etc. Y todas aquellas conductas emitidas en dicha situación se les llama genéricamente 'protooperantes'. Entre las proto-operantes que han sido de particular interés para el AEC está el nivel operante, es decir, aquellas respuestas que el organismo emite sobre la palanca o tecla previamente a la contingencia de reforzamiento (Keller \& Schoenfeld, 1950). El interés de reportar el nivel operante ha sido principalmente para fines de control metodológico (Schoenfeld, Antonitis, \& Bersh, 1950, Segal, 1959; Timberlake, 2004). Sin embargo, una limitación de llamarles proto-elementos de la contingencia es que se asume que dichos elementos serán sometidos a un proceso de condicionamiento.

Una alternativa para describir el efecto conductual de los estímulos, independientemente de un proceso de condicionamiento, es mediante el concepto 'significado conductual' de los estímulos y objetos ambientales (Costall, 2004; Heider, 1959). Este concepto hace referencia a que los organismos perciben las oportunidades de acción que pueden ejecutarse con un objeto, es decir, los objetos constituyen posibilitadores de conducta para los organismos dependiendo tanto de las dimensiones y estructura del objeto como las del organismo (Gibson, 1979; Warren, 1995). Este concepto facilita una descripción conductual de la percepción, aun cuando no se asuma un proceso de condicionamiento a contingencias de reforzamiento. Diferentes investigaciones han mostrado que ciertos patrones de exploración ocurren en función de las posibilidades conductuales de los objetos (Heyser \& Chemero, 2012; Martínez-Escudero, Gámiz, \& Gallo, 2012), incluyendo los objetos propios de una cámara de condicionamiento operante (Cabrera, Sanabria, Jiménez, \& Covarrubias, 2013; Skinner, 1938; 1961; Timberlake, 2004); asimismo, estas conductas según los posibilitadores conductuales de diferentes objetos se han observado en niños en diversidad de procedimientos (Adolph, 1997; Gibson \& Pick, 2000; Ríos-Checa, Quevedo, Ramírez, Gómez-Llanos, \& Jiménez Santa-Cruz, 2010; Tonneau, Kim-Abreu, \& Cabrera, 2004).

Considerando que el AEC, al igual que la aproximación ecológica de la psicología (Gibson, 1966, 1979) se establece como una psicología sustentada en el ambiente y en la relación entre éste y el organismo (Costall, 2004), el presente escrito destaca el concepto de 'posibilitador de conducta' o 'soporte conductual' (affordance) que acuñó Gibson (1979) para describir la propiedad de los objetos para ofrecer y soportar determinadas oportunidades de acción para un organismo en particular. Bajo esta concepción, propiedades del ambiente y del organismo mantienen entre sí una relación de reciprocidad (Costall, 2004; Järvilehto, 1998; Lombardo, 1987; Palmer, 2004) y derivan en el comportamiento del organismo (Warren, 2006). Es bajo esta relación de reciprocidad que el organismo, como ser perceptor y comportante, percibe los objetos ambientales y se comporta ante ellos.

Siendo consecuentes con estos argumentos, y dado que diferentes objetos posibilitan diferentes conductas exploratorias de manera natural (Heyser \& Chemero, 2012; Martínez-Escudero et al., 2012), diversos autores sugieren que para estudiar las posibilidades de acción que ofrecen los objetos, éstos deben ser medidos en relación a la 
estructura y características biomecánicas y dinámicas del sujeto, es decir con una escala definida a partir de las dimensiones corporales, fuerza muscular y flexibilidad articular del sujeto y no con medidas ajenas a la actividad y estructura del sujeto. La primera ha sido llamada métrica intrínseca mientras la segunda ha sido llamada métrica extrínseca (ver Warren, 1995).

La métrica intrínseca implica tomar el sistema de acción del organismo como una unidad natural para medir las propiedades del ambiente, a diferencia de la métrica extrínseca que impone una unidad arbitraria como lo es el sistema métrico decimal. La métrica intrínseca es una escala corporal, incluso una escala conductual, pues por tratarse de actividad y conductas en ejecución, toma en cuenta no sólo aspectos antropométricos sino principios biomecánicos tales como amplitud de movimientos, fuerza, torsión, velocidad, etc. Debido a estas propiedades de la métrica intrínseca es que se puede analizar el ajuste entre el organismo y el ambiente, permitiendo identificar los puntos críticos absolutos o límites máximos en que puede ejecutarse una actividad, así como los puntos óptimos y preferidos por el sujeto en los que dicha actividad es más eficiente, confortable o segura (Warren, 1995 p. 217). El límite crítico absoluto (Warren, 1984; 1995) es aquella propiedad del estímulo (o configuración de estímulos) en relación a ciertas propiedades del organismo que controlan la conducta, indicando los límites y modos en los que una acción puede llevarse a cabo.

Para que la conducta sea adaptable al ambiente, debe ser controlada prospectivamente (von Hofsten, 2004; von Hofsten, Vishton, Spelke, Feng, \& Rosander, 1998), lo cual requiere que los objetos y sus posibilitadores conductuales sean percibidos anticipadamente por el organismo (Turvey, 1992). Es importante señalar que bajo esta perspectiva, la percepción que permite un control prospectivo es conocida como 'percepción directa', es decir, no implica un proceso mental que medie entre el objeto del ambiente y la conducta del organismo (Gibson, 1966, 1979). Este aspecto del control prospectivo y la percepción anticipada de los objetos es compatible con la perspectiva que plantea Skinner (1953) respecto a que "la conducta con la que nos ajustamos al mundo la desarrollamos porque la estimulación visual de un objeto es la ocasión por la cual ciertas respuestas como caminar, alcanzar y otras, conducen a consecuencias táctiles particulares" (p. 108). Por ejemplo, antes de subir un escalón un individuo percibe si la altura del escalón es menor a la longitud de sus piernas (i.e., se encuentra dentro su límite crítico absoluto); asimismo anticipa la postura (modo de acción) que deberá adoptar para ello, dependiendo de la extensión de sus piernas, su flexibilidad y fuerza (Warren, 1984). Todo ello no implica la mediación por parte de una actividad cognitiva que calcule la métrica de cada elemento, sino que la experiencia que se tiene con el tipo de actividad a realizar es determinante para percibir una acción como posible o imposible de realizar (Gibson, 1966; O’Regan \& Nöe, 2001), tal como se ha observado en infantes que gatean y que empiezan a caminar (Adolph, 1997; Adolph, Joh, \& Eppler, 2010).

Diversos estudios han encontrado que sujetos humanos pueden identificar de manera precisa los límites críticos absolutos en diferentes actividades tales como 
CABRERA ET AL.

66

juzgar si es posible pasar a través de una apertura sin rotar los hombros (Chang, Wade, \& Stoffregen, 2009; Warren \& Whang, 1987), si la altura de un escalón permite subirlo utilizando sólo las piernas (Konczak, Meeuwsen, \& Cress, 1992; Warren, 1984), así como juzgar si un objeto colocado sobre una mesa es alcanzable estirando el brazo (Carello, Grosofsky, Reichel, Solomon, \& Turvey, 1989; Mark et al., 1997).

Con el propósito de evaluar el efecto que tiene una de las propiedades de un objeto (i.e. la altura del estímulo) respecto a las posibilidades de acción que ofrece a un sujeto, se utilizó una tarea en la que los participantes debían estimar si podían alcanzar una serie de líneas proyectadas en una pantalla a diferentes alturas, ya sea utilizando sus propios brazos (Experimento 1) o con un objeto ajeno a ellos (Experimento 2).

Dado que las posibilidades de acción que un objeto ofrece son relativas a diversas características de un sujeto, tales como su estructura, posición, postura, antropometría, etc. (Gibson, 1979; Warren, 1995), en los experimentos 1 y 2 se evaluó la respuesta de los participantes utilizando dos soportes corporales de diferente tamaño (silla pequeña y silla alta), con lo cual se contrastó la percepción de las posibilidades de acción del estímulo según dos alturas diferentes. Con los dos soportes corporales se altera el ángulo visual que los participantes tienen para cada estímulo (Bertami, Yang \& Proffitt, 1998), con lo cual se espera que, bajo una métrica extrínseca (ver arriba), la posibilidad de acción de los estímulos difiera según la altura del soporte corporal (sillas alta y pequeña), pero transformando a una métrica intrínseca, las posibilidades de acción se igualarán para ambos soportes.

\section{Experimento 1}

\section{Método}

Participantes. Participaron 12 estudiantes de la Licenciatura en Psicología de la Universidad de Guadalajara (ocho mujeres y cuatro varones) con agudeza visual normal y edades con un rango de 21 a 26 años. Todos los participantes eran diestros.

Aparatos y situación experimental. El experimento se llevó a cabo en un cubículo de $5 \times 4 \mathrm{~m}$, con luz atenuada con el objetivo de que la imagen proyectada pudiera ser vista con facilidad. Se utilizó un proyector de imágenes BENQ ${ }^{\circledR}$ para presentar los estímulos, una computadora portátil HP, hojas de registro, una cinta métrica adherida a un cortinero metálico ajustable que se utilizó como antropómetro y dos sillas, una con el asiento a una altura de $30 \mathrm{~cm}$ respecto al piso y otra con altura de $74 \mathrm{~cm}$. La distancia entre la pared en donde se proyectó la imagen y la silla en donde se sentaron los participantes fue de $250 \mathrm{~cm}$.

Procedimiento. El experimento consistió en presentar secuencialmente una serie de 78 líneas horizontales proyectadas en la pared frontal a diferentes alturas respecto al piso. Cada línea proyectada en la pared definió un ensayo. El participante tenía que decir en cada ensayo si la línea proyectada podía ser alcanzada o tocada por él, su- 
poniendo que se encontrara justo debajo de la línea proyectada. Además tenía que decir qué tan seguro estaba de su respuesta. Para ello se le preguntaba "¿Cuánto apostarías por tu respuesta?", en donde el rango de apuesta iba de $\$ 0$ a $\$ 600$ con incrementos de $\$ 100$. Se utilizó esta forma de apuesta ficticia para evaluar la seguridad de sus respuestas por haber observado, en procedimientos piloto, que los sujetos se desempeñaban mejor bajo este modo que si se utilizaba una escala tipo Likert para estimar qué tan seguros estaban de su respuesta. El rango es arbitrario, pero incluyendo el valor de \$0 para tener la posibilidad de máxima inseguridad del participante. La progresión (incrementos de \$100) y el máximo (\$600) se decidió con base a tener mayor precisión en esta variable, de al menos siete valores posibles de respuesta. El rango de alturas de las líneas fue de 100 a $254 \mathrm{~cm}$, con una diferencia entre cada altura de dos centímetros. El orden de presentación de los ensayos (diferentes alturas) fue aleatorio. Cada participante respondió a los 78 ensayos en dos condiciones experimentales, sumando un total de 156 ensayos. En la condición de soporte bajo el participante se sentó en la silla de $30 \mathrm{~cm}$. En la condición soporte alto el participante respondió sentado en la silla de $74 \mathrm{~cm}$.

Los 78 ensayos presentados para cada altura de la silla se dividieron en tres bloques de 26 ensayos cada uno, con el objetivo de proporcionar entre cada bloque un período de descanso de dos minutos, opcional para el participante. Cada ensayo duró lo que le requirió al participante expresar verbalmente su respuesta "si" o "no" y decir qué tan seguro estaba de su respuesta; el experimentador anotaba la respuesta del participante en las hojas de registro y después de ello se proyectaba la línea del siguiente ensayo. Al término de cada ensayo, la computadora emitía un breve sonido agudo (beep) con el objetivo de señalar al participante que en seguida aparecería una nueva línea. El orden de las condiciones fue contrabalanceando; algunos participantes iniciaron en la condición de soporte alto y otros iniciaron en la condición de soporte bajo.

Se pidió a los participantes que se sentaran recargando su espalda erguida en el respaldo y colocaran las manos sobre sus muslos. Una vez sentados en la posición correcta, el experimentador leyó y mostró a los participantes las instrucciones de la tarea proyectadas en la pared:

¡Bienvenido(a)! Te pedimos que recargues completamente tu espalda sobre el respaldo de la silla y que permanezcas con las manos sobre tus piernas.

A continuación se te presentará una serie de líneas horizontales. Cuando la posición de cada línea cambie escucharás un sonido. Tu tarea consiste en imaginar que así como estás sentado(a) alzas tu brazo derecho para alcanzar las líneas. Imagina que las líneas son cuerdas de un instrumento musical a las cuales harás sonar. El sólo rozarlas es suficiente para decir que son alcanzables. Tú nos dirás la palabra 'Sí', si crees que a esa altura pudieras alcanzar a tocar la cuerda con tus dedos, o por el contrario nos dirás 'No' si crees que no la alcanzarías aún extendiendo tu brazo al máximo. Procura responder lo más exacto que puedas. 
Después de decir si alcanzas o no alcanzas la cuerda, deberás decir cuánto apostarías (de manera ficticia) por tu respuesta: $\$ 0, \$ 100, \$ 200, \$ 300, \$ 400$, $\$ 500$ o $\$ 600$. Cuanto más apuestes es porque más seguro te encuentras de tu respuesta.

Si tienes alguna duda puedes preguntarla ahora.

¡Muchas gracias!

Inmediatamente después de mostrar las instrucciones se utilizó un ensayo de ejemplo para asegurar que el participante había comprendido las instrucciones de la tarea. Si no había dudas por parte del participante, daba inicio el experimento.

Medición antropométrica. Una vez terminados todos los ensayos, se le pidió al participante que permaneciera en el lugar para tomar algunas medidas corporales. Con la cinta métrica adherida a la barra metálica se tomaron las siguientes medidas corporales: a) longitud del brazo derecho en posición horizontal, definida como la distancia desde el acromion (hueso que forma la cresta superior del hombro) hasta la punta del dedo medio, b) altura al hombro, definida como la longitud desde el piso hasta el acromion del hombro derecho estando el participante en posición sedente $y$, c) alcance máximo vertical, definido como la distancia del piso a la punta del dedo medio del brazo derecho extendido hacia arriba, según la indicación de "tocar lo más arriba que te sea posible" con el participante en posición sedente. Las medidas b y c fueron tomadas al participante sentado en ambas alturas de las sillas.

Análisis de datos. Para analizar la percepción de la alcanzabilidad del estímulo, por tratarse de una variable dicotómica la respuesta que daban los participantes (respuestas sí o no), se asignó valor de 1 a las respuestas 'sí', y valor de 0 a respuestas 'no'. Con estos valores se tomó el promedio móvil de tres alturas contiguas. Dichos valores reflejaron la proporción de respuestas 'sí alcanzo' en valores entre 0 y 1 .La razón de tomar el promedio móvil de cada tres alturas contiguas fue que, en procedimientos piloto y con datos anecdóticos los sujetos reportaron las tres alturas contiguas como la misma altura.

Para analizar la seguridad de su respuesta, a cada cantidad de la apuesta ficticia $(\$ 0, \$ 100, \$ 200 \ldots$... \$600 pesos), se asignó un valor proporcional respecto al máximo (\$600), en el que el mínimo era 0 y el máximo (\$600) tomó un valor de 1.0. Al igual que en la variable de percepción de la alcanzabilidad, se tomó el promedio móvil de tres alturas contiguas.

Resultados y discusión. La Figura 1a muestra la proporción promedio de ocasiones en las que los participantes respondieron 'sí alcanzo' al estímulo proyectado en la pared en función de la altura de dicho estímulo. Los círculos llenos representan la condición de soporte bajo (silla de $30 \mathrm{~cm}$ ) y los círculos vacíos representan la condición soporte alto (silla de $74 \mathrm{~cm}$ ). En la condición de soporte bajo, el punto de indiferencia (valor de 0.5 en el eje de la ordenada) en la estimación del estímulo como alcanzable o inalcanzable correspondió a la altura de $180 \mathrm{~cm}$, mientras que en la condición de soporte alto, el punto de indiferencia se ubicó a la altura de $215 \mathrm{~cm}$. En la condición 

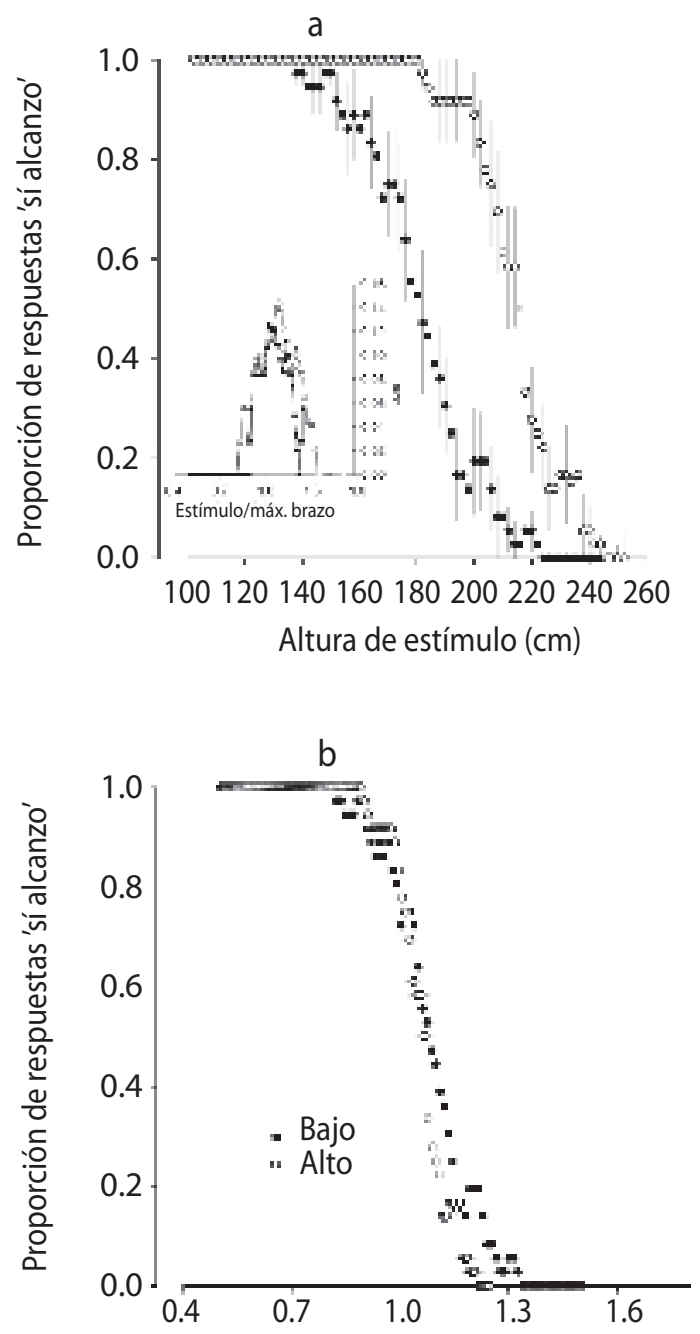

Altura de estímulo / alcance máx. de brazo

Figuras 1a y 1b: Proporción promedio de respuestas como estímulo alcanzable en función de la altura de las líneas proyectadas (Figura 1a) y en función de la métrica intrínseca expresada como la razón entre la altura del estímulo sobre el alcance máximo vertical de los sujetos (Figura 1b). Cada punto representa los datos promedio de todos los participantes y las líneas de error muestran el error estándar a la media. El gráfico insertado en Figura 1a muestra la distribución del error estándar de las respuestas como estímulo alcanzable en términos de la métrica intrínseca. Ver texto principal para mayores detalles. 

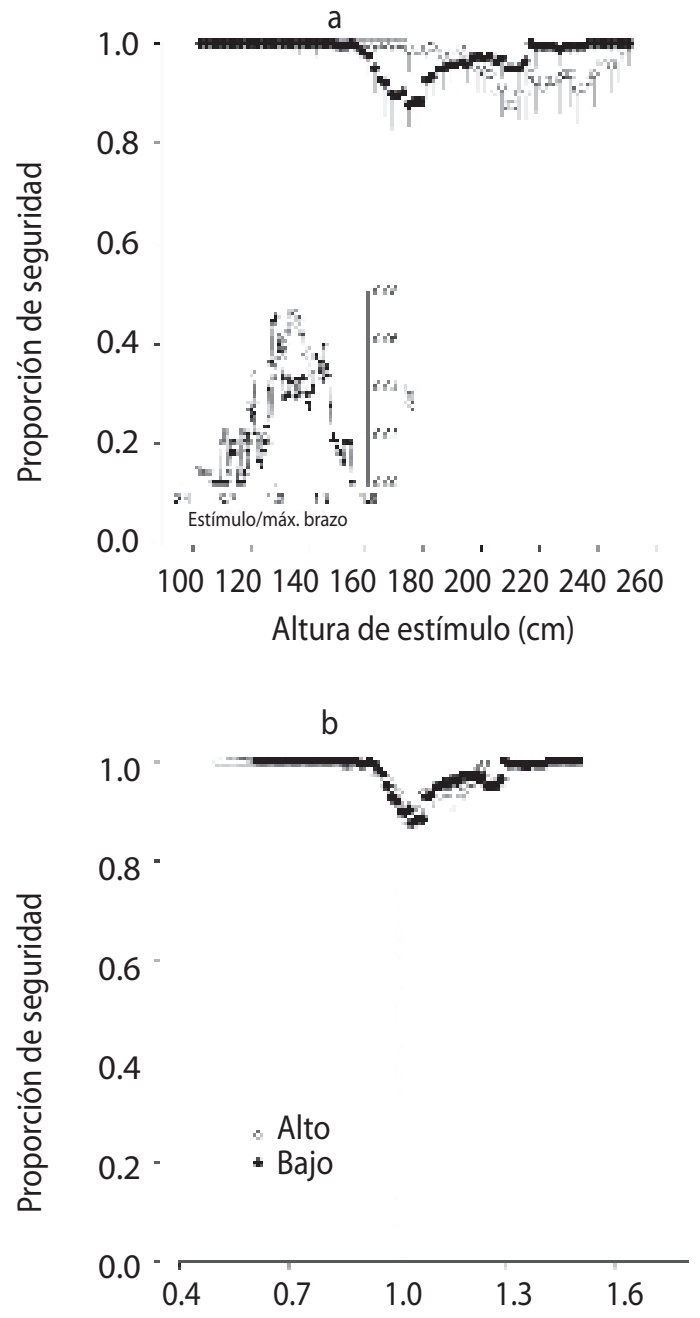

Altura de estímulo / alcance máx. de brazo

Figuras 2a y 2b: Proporción promedio de seguridad con la que los sujetos emitían sus estimaciones en función de la altura de las líneas proyectadas (Figura 2a) y en función de la métrica intrínseca (Figura 2b). Cada punto representa los datos promedio de todos los participantes y las líneas de error muestran el error estándar a la media. El gráfico insertado en Figura 2a muestra la distribución del error estándar de la seguridad de las respuestas en términos de la métrica intrínseca. 
soporte bajo ningún participante respondió al estímulo como alcanzable a la altura arriba de $220 \mathrm{~cm}$, mientras que en la condición soporte alto, los participantes consideraron como inalcanzable el estímulo a una altura por encima de $240 \mathrm{~cm}$. El Apéndice A muestra los datos individuales para las condiciones soporte bajo (círculos grises) y soporte alto (rombos vacíos).

La seguridad que los participantes manifestaron tener respecto a su estimación sobre si el estímulo era alcanzable o inalcanzable se muestra en la Figura 2a en función de la altura del estímulo. En general, los participantes mostraron seguridad en sus estimaciones. En la condición de soporte bajo (círculos llenos) la seguridad de su respuesta cayó a un mínimo de 0.87 que correspondió a estímulos con alturas entre 170 y $180 \mathrm{~cm}$, mientras que en la condición de soporte alto (círculos vacíos), los participantes manifestaron una seguridad mínima también de 0.87 pero ante estímulos ubicados a la altura entre 206 y $212 \mathrm{~cm}$. En el Apéndice B se muestran los datos individuales de los índices de seguridad para las condiciones soporte bajo (círculos grises) y soporte alto (rombos vacíos).

Para describir la altura de las líneas en términos del significado conductual de 'alcanzabilidad' por parte de los participantes, siendo consistentes con la propuesta ecológica de Gibson (1979), se realizó el análisis desde la métrica intrínseca descrita por Warren (1995) y se representó en las figuras 1 b y 2b. En este análisis, el eje de la abscisa constituye el cociente de la altura del estímulo sobre el alcance máximo vertical. Una estimación perfecta del límite crítico absoluto (Warren, 1984) o identificación de la altura en la que el estímulo deja de ser alcanzable y se convierte en inalcanzable mostraría la curva en valores de 1.0 desde la altura 0.4 hasta la altura 1.0 (eje de la abscisa), adquiriendo valores de 0.0 a partir de la altura 1.01 (i.e., a partir de la primera altura que sobrepasa el límite crítico absoluto). Un desplazamiento de la curva a la derecha o a la izquierda representa, respectivamente, una sobreestimación o una subestimación de la capacidad de alcance del participante determinada por la dimensión corporal del alcance máximo vertical.

La Figura 1 b muestra que la estimación de la altura del estímulo como alcanzable o inalcanzable se superpone en ambas condiciones, soporte alto (círculos vacíos) y soporte bajo (círculos llenos), teniendo que el punto de indiferencia en la estimación de alcanzable o inalcanzable se ubica encima de 1.10, es decir, los participantes tendieron a sobreestimar ligeramente su capacidad de alcance en ambas condiciones, siendo un poco más precisos en el asiento alto que en el asiento bajo. El Apéndice $C$ muestra los datos individuales utilizando la métrica intrínseca, se observa que en la mayoría de los participantes ambos grupos de símbolos están superpuestos. La tendencia a sobreestimar las capacidades de alcance es un resultado consistente con estudios previos que evaluaron estimaciones de alcance de objetos que se encontraban a diferentes distancias sobre una mesa (Carello et al., 1989; Mark et al., 1997; Rochat, 1995).

Insertado en la Figura 1a se muestra el gráfico correspondiente al error estándar respecto al promedio en función del cociente altura del estímulo sobre el alcance máximo vertical (métrica intrínseca). Éste gráfico muestra que el error estándar para 
las dos condiciones tiene la misma distribución, con el error más alto en el valor cercano a 1.0, es decir, mayor divergencia en las respuestas en los valores cercanos al límite crítico absoluto.

Utilizando la métrica intrínseca para analizar la seguridad con la que los participantes estimaron el alcance con su brazo, la Figura $2 \mathrm{~b}$ muestra en general una superposición entre las condiciones de soporte alto (círculos vacíos) y soporte bajo (círculos llenos), con los menores índices de seguridad cercanos al límite crítico absoluto, con un ligero desplazamiento a la derecha, correspondiente con la sobreestimación de los participantes mostrada en la Figura 1 b (el Apéndice D muestra los datos individuales de la métrica intrínseca). Insertado en la Figura 2a se muestra el gráfico con el error estándar de la certeza con la que los participantes estimaron al estímulo como alcanzable o inalcanzable. La distribución del error es similar en ambas condiciones, sin embargo la condición soporte bajo (círculos llenos) muestra una distribución bimodal.

En general ambas medidas, la estimación de alturas alcanzables y la seguridad en sus estimaciones, muestran consistencia entre sí, en donde la menor seguridad que los participantes tuvieron en sus estimaciones y la mayor divergencia en sus respuestas de estimación (error estándar) ocurrieron muy próximos al límite crítico absoluto. Resultados similares fueron obtenidos en experimentos en los que los participantes estimaron si podían subir escalones de diferentes alturas (Warren, 1984) o pasar a través de aberturas sin rotar los hombros (Warren \& Whang, 1987).

\section{Experimento 2}

El análisis desde la métrica intrínseca surge bajo el supuesto de que un individuo, al percibir una propiedad del ambiente, puede anticipar una acción en función de su propia estructura y biomecánica (Warren, 1995), dicho de otro modo, permite evaluar en qué medida ciertos elementos del campo visual son la ocasión para realizar una acción efectiva (Skinner, 1953). En el Experimento 1 se mostró que los participantes ajustaron su respuesta en función de los cambios en la altura del soporte corporal (i.e., la altura del asiento), con una ligera sobreestimación en sus juicios de alcance de las líneas proyectadas. Evidencia experimental (Marotta \& Goodale, 2001; Schiffman, 1965) sugiere que los individuos estiman longitudes y tamaños de manera más precisa utilizando unidades de medida familiares que utilizando unidades de medida novedosas o poco familiares. Por ejemplo, Schiffman (1965) evaluó la precisión con la que sus participantes estimaron la longitud de líneas de diferente longitud dibujadas en una hoja. Encontró que la unidad de medición más familiar para cada sujeto (centímetros o pulgadas) favoreció una mayor precisión que cuando se evaluó con una unidad poco familiar. Con otro procedimiento, Rochat (1995) pidió a participantes que estimaran si ellos mismos, así como otro individuo, alcanzaban un objeto a diferentes distancias. Cuando la estimación se hizo a partir de si ellos mismos alcanzaban el objeto, los participantes fueron más precisos que cuando la estimación se 
hizo respecto a si otra persona alcanzaba el objeto. A partir de estos hallazgos surge la siguiente pregunta: si los participantes utilizaran un objeto ajeno al cuerpo, y por lo tanto menos familiar que la extensión del propio brazo, para estimar a qué altura las líneas son alcanzables ¿cambiará el ajuste en su estimación respecto al límite crítico absoluto? La pregunta es pertinente en numerosas situaciones de la vida cotidiana, pues muchas veces un objeto se vuelve una "extensión" o "sustituto" de la propia unidad funcional brazo-antebrazo-mano, por ejemplo, pegarle a una piñata usando un palo o cortar fruta de una rama usando un gancho. Para ello, la percepción de la posibilidad de acción debe definirse a partir de la medida de un objeto ajeno al cuerpo. Para responder a esta pregunta, el Experimento 2 consistió en evaluar la estimación que los participantes hicieron respecto a si un objeto (una barra de longitud ajustable) podía tocar las líneas proyectadas sobre una pantalla, en donde las dimensiones de la barra correspondieron a la altura máxima alcanzable por los participantes con su brazo extendido (alcance máximo vertical) en dos alturas de soporte, Soporte Alto y Soporte Bajo, tal como en el Experimento 1.

\section{Método}

Participantes. Participaron 16 estudiantes de la licenciatura en psicología de la Universidad de Guadalajara, ocho mujeres y ocho varones, con un rango de edad de 18 a 31 años, todos los participantes eran diestros y tenían agudeza visual normal.

Aparatos y situación experimental. Se utilizó un proyector de imágenes $B E N Q^{\circledR}$, una computadora portátil mini $\mathrm{HP}^{\circledR}$, una pantalla blanca $3 \mathrm{M}^{\circledR}$ en donde se proyectaron las imágenes, una barra de aluminio de longitud ajustable, un antropómetro tipo Harpenden (Holtain Ltd., Reino Unido) y un flexómetro. Como soportes alto y bajo se utilizaron una silla de $39 \mathrm{~cm}$ de altura y un escritorio de $75.1 \mathrm{~cm}$ de altura. La pantalla estuvo colocada a una distancia de $250 \mathrm{~cm}$ respecto al participante.

Procedimiento. El procedimiento fue igual que en el Experimento 1, excepto que las medidas antropométricas (ver abajo) fueron tomadas previo al inicio de la sesión para poder ajustar la longitud de la barra a la dimensión del alcance máximo vertical de los participantes. El experimentador dio al participante un barra ajustable y se le pidió que observara con detenimiento, principalmente su longitud, pero siempre en posición horizontal. Los participantes sostuvieron la barra con sus manos sobre sus muslos durante todo el experimento con el objetivo de que pudieran observarla en cualquier momento, pero con la instrucción de no cambiarla a posición vertical.

Las instrucciones fueron las siguientes:

¡Bienvenido! Seguramente en este momento ya te encuentras sentado. Ahora te pedimos que recargues completamente tu espalda sobre el respaldo de la silla y permanezcas con las manos sobre tus muslos.

Observa bien el tubo que te mostrará el experimentador. Sostenlo con tus manos sobre tus muslos sin moverlo. 
Enseguida se te presentará una serie de líneas proyectadas en la pantalla. Cuando cambie la línea escucharás un sonido. Imagina que el tubo se encuentra verticalmente parado sobre el piso y recargado en la pantalla. Tu trabajo consiste en decirnos si el tubo alcanzaría a tocar las líneas horizontales. El solo hecho de rozarlas es suficiente para decir que sí alcanza a tocarlas.

Una vez que estés seguro de si el tubo puede tocar o no las líneas, debes decirlo en voz alta. Procura responder lo más exacto que puedas.

Después de decir si el tubo alcanza o no la línea, deberás decir cuánto apostarías por tu respuesta: $\$ 0, \$ 100, \$ 200, \$ 300, \$ 400$, $\$ 500$ o $\$ 600$. Obviamente, cuanto más apuestes, es porque más seguro estás de tu respuesta.

Se presentaron un total 140 ensayos: 70 ensayos fueron presentados en la condición de soporte alto (sentados sobre el escritorio) y 70 ensayos en la condición de soporte bajo (sentados en la silla). Se contrabalanceó el orden de condiciones presentadas, iniciando ocho participantes en la condición soporte alto y los otros ocho iniciando en la condición soporte bajo.

Medición antropométrica. Con el antropómetro se tomaron las siguientes medidas corporales a los participantes antes de iniciar el experimento, para lo cual se les pidió sentarse en la silla con soporte bajo: a) alcance máximo vertical, definido al igual que en el Experimento 1 como la longitud desde el piso hasta la punta del dedo medio del brazo derecho extendido hacia arriba según la indicación de "tocar lo más arriba que te sea posible"; b) altura a los ojos, esto es, la distancia del piso a la pupila del ojo derecho; y c) estatura sentado, la altura desde el piso hasta el extremo superior de la cabeza (vertex). La primera medida fue utilizada para ajustar la barra a esa longitud correspondiente a la condición soporte bajo; para la condición soporte alto, a esta medida se le sumó la diferencia $(36.1 \mathrm{~cm})$ entre ambos suportes, y con ello se ajustó la longitud de la barra. De este modo, las longitudes de la barra en las condiciones soporte bajo y soporte alto fueron idénticas al alcance máximo vertical de los participantes sentados en el soporte bajo y el soporte alto, respectivamente. Las medidas antropométricas b) y c) se tomaron sólo para distraer al participante y evitar, en la medida de lo posible, que dedujera que de la primera medida se ajustaría el tamaño de la barra, y tratar de impedir que respondiera a la altura de los estímulos según si él o ella alcanzara con su brazo las líneas, tal como en el Experimento 1.

\section{Resultados y Discusión}

La Figura 3a muestra la proporción promedio de respuestas en que los participantes consideraron que la barra alcanzaba a tocar los estímulos en función de la altura de los mismos para la condición soporte bajo (círculos llenos) y la condición soporte alto (círculos vacíos). La proporción de respuestas afirmativas decreció en función de la altura de los estímulos, teniendo el punto de indiferencia la condición soporte bajo a una altura de $180 \mathrm{~cm}$ y la condición de soporte alto cerca de $200 \mathrm{~cm}$. 
La seguridad que manifestaron tener los participantes en su estimación se calculó igual que en el Experimento 1, y se muestra en la Figura 4a como una función de la altura del estímulo. La mínima seguridad en la condición soporte bajo fue cerca de 0.65 entre las alturas 182 y $186 \mathrm{~cm}$, mientras que en el soporte alto esta mínima seguridad fue de 0.62 entre las alturas de 208 a $212 \mathrm{~cm}$.

La Figura 3b muestra la estimación del alcance de la barra en las líneas proyectadas en términos de métrica intrínseca. Se observa en los participantes una tendencia a subestimar la altura de la barra, y en mayor medida en la condición soporte alto, ya que a pesar que los puntos de indiferencia (0.5) en su estimación cayeron cerca del límite crítico absoluto (valor en abscisas de 1.0), desde alturas entre 0.6 y 0.8 en diversos ensayos hubo estimaciones de que la barra no alcanzaría a tocar al estímulo (el Apéndice E muestra los datos individuales). Este resultado es similar al encontrado por Rochat (1995), quien en dos condiciones acomodó un objeto a diferentes distancias (condición horizontal) y a diferentes alturas (condición vertical), y pidió a niños y a adultos que estimaran la capacidad de alcanzarlo por parte del propio experimentador. Los resultados mostraron que los niños al igual que los adultos subestimaron las capacidades de alcance del experimentador tanto en el plano horizontal como en el plano vertical.

Respecto a la seguridad de sus respuestas, la Figura $4 \mathrm{~b}$ muestra que los participantes dudaron más de sus respuestas en valores cercanos al límite crítico absoluto (abscisas en 1.0), con un ligero desplazamiento a la derecha en la condición soporte bajo (círculos llenos). En el Apéndice F se muestran los datos individuales de la métrica intrínseca para esta variable dependiente. Insertas en las figuras 3a y 4a se muestran las gráficas de las distribuciones del error estándar para las estimaciones y para la seguridad de las mismas respectivamente. En ambos gráficos se observa que los mayores valores de la variabilidad se encontraron cercanos al límite crítico absoluto, resultado consistente con el del Experimento 1.

Finalmente, con el objetivo de comparar el ajuste que los participantes hicieron en sus estimaciones de la altura de los estímulos con sus brazos o con la barra, en la Figura 5 se muestran las curvas correspondientes al Experimento 1 (símbolos llenos) y Experimento 2 (símbolos vacíos) para ambas condiciones. Se ajustó una función logística con coeficiente negativo ${ }^{1}$ para evaluar la precisión en la discriminación de los estímulos como alcanzables o inalcanzables (líneas continuas). En una discriminación perfecta las líneas descenderían verticalmente (línea punteada) teniendo el punto de indiferencia en 1.0 (eje de la abscisa) y el índice de decremento tendería a cero; cuanto más difiera de cero menor precisión en la discriminación. La distribución de respuestas como "estímulo alcanzable" con el propio brazo de los participantes (Experimento 1) tuvo mayor precisión con valores absolutos en los índices de

\footnotetext{
${ }^{1}$ Para fines analíticos, la función logística utilizada tiene la forma $\mathrm{A} /\left(1-\mathrm{Be}^{\left.-{ }^{k t}\right)}\right.$ en donde $\mathrm{A}$ es la máxima proporción alcanzable (valor fijo a 1.0); B es el punto de indiferencia que indicaría sobreestimación cuando $\mathrm{B}>0$ y subestimación cuando $\mathrm{B}<0$. El valor $\mathrm{k}$ indica la tasa de cambio, en nuestro análisis corresponde a la discriminabilidad entre alcanzable o inalcanzable, cuanto más cercano a cero, mayor precisión en la discriminación (su valor es negativo dada la forma decreciente de la función).
} 
CABRERA ET AL.

a

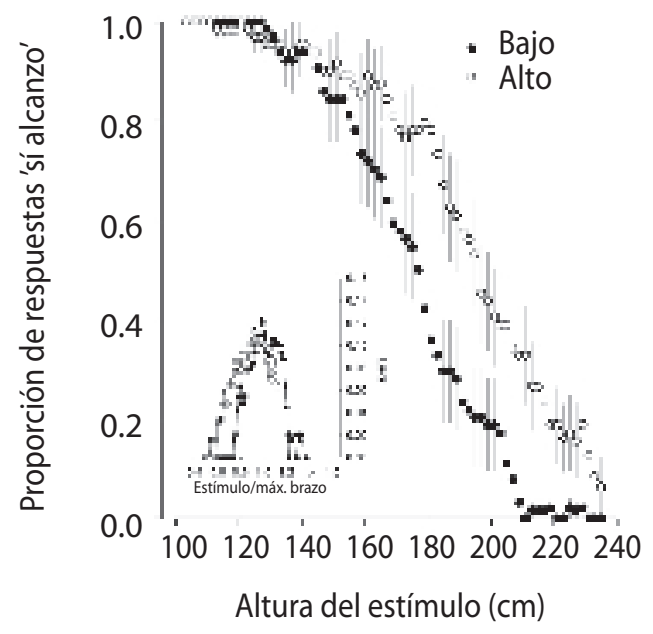

b

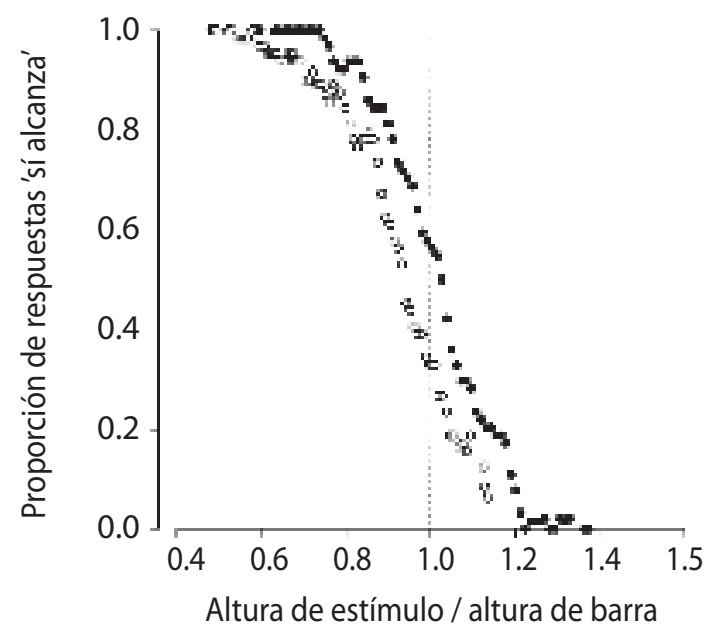

Figuras 3a y 3b: Proporción promedio de respuestas como estímulo alcanzable por la barra en función de la altura de las líneas proyectadas (Figura 3a) y en función de la métrica intrínseca tomando la razón entre la altura del estímulo sobre la longitud de la barra (Figura 3b). Cada punto representa los datos promedio de todos los participantes y las líneas de error muestran el error estándar a la media. El gráfico insertado en Figura 3a muestra la distribución del error estándar de las respuestas como estímulo alcanzable por la barra en términos de la métrica intrínseca. 

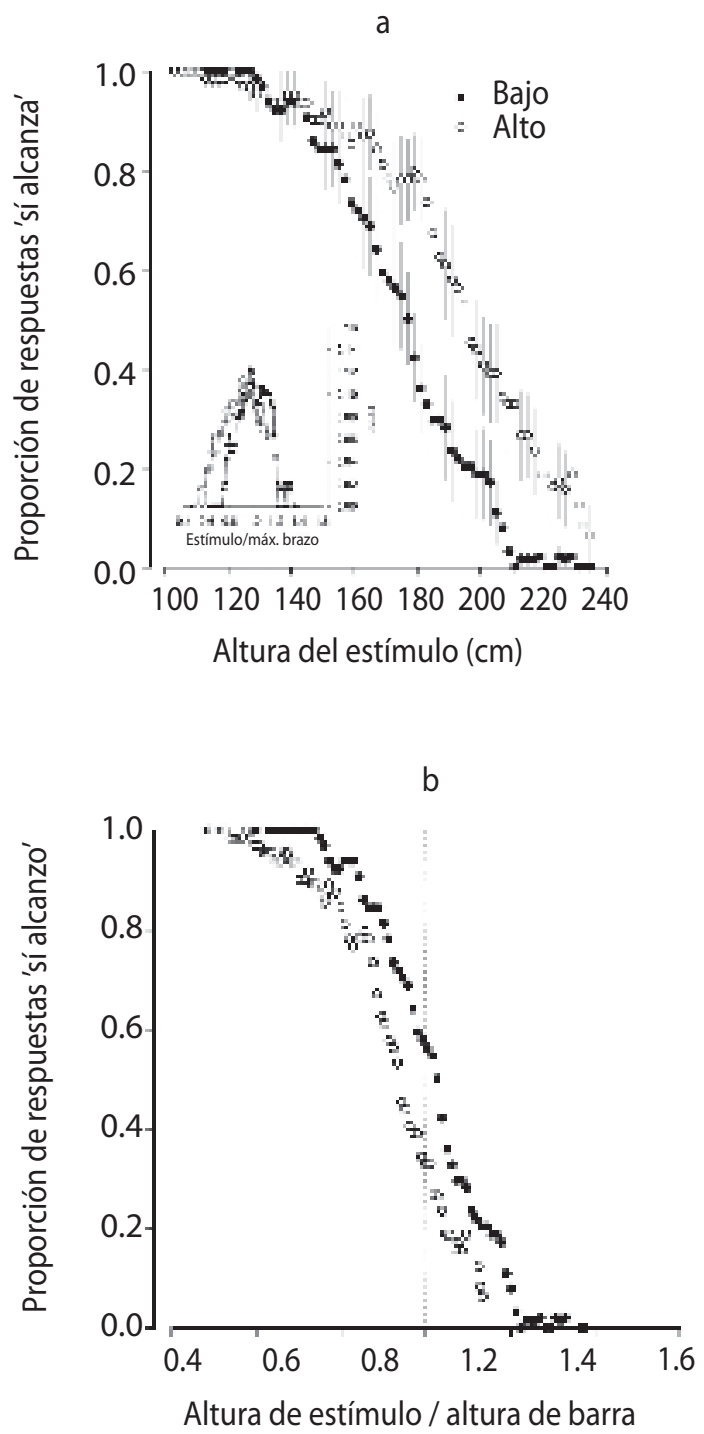

Figuras 4a y 4b: Proporción promedio de seguridad con la que los sujetos emitían sus estimaciones en función de la altura de las líneas proyectadas (Figura 4a) y en función de la métrica intrínseca (Figura 4b). Cada punto representa los datos promedio de todos los participantes y las líneas de error muestran el error estándar a la media. El gráfico insertado en Figura 4a muestra la distribución del error estándar de la seguridad de las respuestas en términos de la métrica intrínseca. 


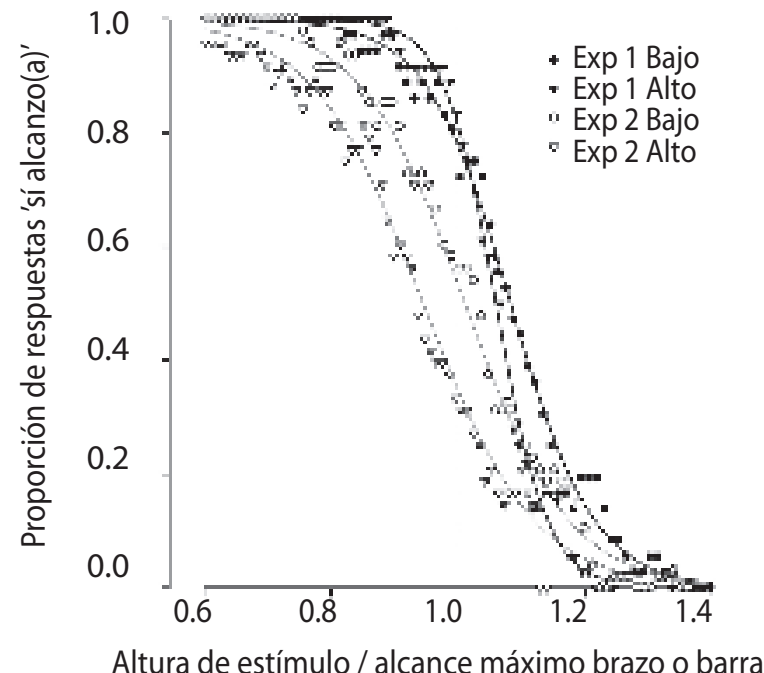

Figura 5. Comparación de los promedios de respuestas como estímulo alcanzable con el brazo (Experimento 1) y con la barra (Experimento 2) en ambas alturas de soporte, en función de la métrica intrínseca del estímulo. Ver texto principal para mayores detalles.

descenso de 0.06 (soporte bajo) y 0.04 (soporte alto), que la estimación hecha con un objeto externo (Experimento 2) con valores absolutos en los índices de descenso de 0.08 para ambas condiciones (soportes bajo y alto), ello a pesar de que la longitud de la barra fue idéntica a su alcance máximo vertical (i.e., la altura máxima que los participantes alcanzarían con su brazo extendido hacia arriba). Estos resultados son compatibles y complementarios con hallazgos experimentales previos (Burton \& Cyr, 2004; Rochat, 1995; Schiffman, 1965) que muestran que los sujetos estiman longitudes de manera más precisa utilizando unidades de medida familiares (i.e., medidas de su propio cuerpo) que utilizando unidades novedosas como barras de diferentes longitudes o la longitud del brazo de otra persona. Asimismo, estos resultados sugieren que la estimación perceptual basada en las capacidades corporales es más precisa que la estimación basada en un objeto porque las condiciones naturales del ambiente generalmente demandan al organismo una estimación con base en sus propias capacidades de acción.

\section{Discusión general}

Los resultados del Experimento 1 mostraron que los participantes fueron precisos para estimar sus propias capacidades de alcance señalando a qué alturas la línea horizontal era alcanzable o inalcanzable para ellos, mostrando una mayor cantidad de 
errores en sus estimaciones en alturas cercanas al límite crítico absoluto, resultado consistente con diversos estudios (e.g., Warren, 1984; Warren \& Whang, 1987). Por ejemplo, en un experimento Warren (1984) mostró a sus participantes fotografías de escaleras de diferentes alturas, y pidió a los participantes que respondieran si era posible subirlas bípedamente (sin utilizar los brazos para subir a ellas). Dividió en dos grupos a sus participantes según la estatura (talla pequeña y talla alta). Warren encontró que con la métrica intrínseca la estimación de los participantes respecto a si podían o no subir los escalones se ajustó a la altura de los sujetos, no obstante los sujetos también mostraron mayor incertidumbre en sus respuestas y mayor variabilidad en alturas cercanas al límite crítico absoluto. En conjunto con los hallazgos de Warren, los resultados del presente estudio sugieren que los sujetos percibieron sus propias limitaciones de acción con base en la relación recíproca entre sus propiedades corporales (i.e., su alcance máximo vertical) y las características del ambiente (i.e., la altura de la línea horizontal), relación mostrada en los análisis de métrica intrínseca.

Al aplicar una métrica intrínseca a la relación entre las dimensiones del ambiente y las dimensiones del organismo, las diferencias observadas entre las condiciones de soporte bajo y soporte alto expresadas en unidades arbitrarias (i.e., $\mathrm{cm}$ ) se minimizaron, es decir, las curvas tendieron a superponerse, indicando que las estimaciones estuvieron bajo el control de la altura del soporte en interacción con la longitud del brazo (Experimento 1) y la longitud de la barra (Experimento 2). Este resultado coincide con estudios que evaluaron estimaciones perceptuales para acciones de alcanzar objetos, subir escalones y pasar por aberturas (Carello et al., 1989; Rochat, 1995; Warren, 1984; Warren \& Whang, 1987). Un hallazgo importante fue que la precisión en la discriminación de "alcanzar" el objeto, así como la seguridad de su estimación, fue mayor cuando se estimó a partir de la propia extremidad del participante (Experimento 1) que con un objeto externo (Experimento 2) a pesar de que la longitud de dicho objeto externo era idéntica a la altura máxima que alcanzarían los participantes con su brazo extendido en posición vertical.

Aunque las estimaciones de los participantes en ambos experimentos fueron precisas, a alturas cercanas al límite crítico absoluto los participantes del Experimento 1 tendieron a sobreestimar ligeramente su alcance máximo vertical, y los participantes del Experimento 2 tendieron a subestimar el alcance de las barras; de manera interesante, el primer hallazgo coincide con otros estudios en que los participantes reportaron que podían alcanzar con sus propios brazos un objeto en el plano horizontal a distancias en las que ya no era posible alcanzarlo sin cambiar su postura (Carello et al., 1989; Mark et al., 1997; Rochat, 1995); en contraste, cuando la estimación consistió en responder si otro sujeto podía alcanzar un objeto, los sujetos tendieron a subestimar su posibilidad de alcance (Rochat, 1995), compatible con el resultado obtenido en el Experimento 2 del presente estudio.

Sobre la tendencia a sobreestimar las propias capacidades de alcance, algunos autores han señalado que en situaciones de la vida cotidiana, si un sujeto se equivoca al estimar una distancia para alcanzar un objeto, puede fácilmente corregirlo agre- 
CABRERA ET AL.

gando más grados de libertad a su movimiento, es decir, involucrando más grupos musculares a la acción de alcanzar (ver Carello et al., 1989). Al respecto, otros autores (Heft, 1993) han sugerido que es la propia tarea experimental la que provoca este tipo de errores al convertir una actividad no reflexiva (alcanzar un objeto con el brazo), en una tarea reflexiva al preguntarle al sujeto si piensa que puede alcanzar un objeto con el brazo. De acuerdo a esta interpretación, Heft (1993) pidió a sujetos que estimaran si podían alcanzar un objeto colocado sobre una mesa con la finalidad de usar esta estimación para completar otra actividad (unir puntos con líneas que tendrían la misma longitud estimada por los sujetos). De esta manera, en el estudio de Heft la estimación de la distancia dejó de ser la tarea principal y se convirtió en una tarea secundaria que tenía un propósito. Sus resultados mostraron que la estimación de las distancias fue precisa, esto es, los sujetos no sobreestimaron sus capacidades de alcance.

Es probable que el resultado de Heft se deba a que un error en la estimación de la distancia conducía a que el sujeto no completara la tarea de manera correcta, lo cual podría interpretarse como una consecuencia aversiva. Evidencia experimental que apoya esta aseveración sugiere que cuando los errores en los juicios en la estimación de las distancias pueden conducir a eventos peligrosos como caerse al cruzar una hendidura, la estimación perceptual es precisa y los sujetos subestiman en lugar de sobreestimar sus capacidades motoras (Jiang \& Mark, 1994). Por otra parte, evidencia experimental en procedimientos de aprendizaje perceptual ha mostrado que con el entrenamiento, los sujetos logran ejecuciones precisas en tareas en las que se requería estimar la distancia a la que se encontraban diferentes objetos a partir de transformaciones en el tamaño de dichos objetos asociados a cambios en el ángulo visual (Epstein, 1967). Con base en lo anterior, es posible que introduciendo una contingencia, tal como retroalimentar la estimación correcta respecto al propio límite absoluto, pueda incrementarse la precisión en la estimación, así como la seguridad que los sujetos tienen de su estimación, lo cual representaría de manera más precisa las condiciones naturales en las que la percepción-acción ocurre, es decir, la contingencia sensoriomotora del sistema óculo-músculo-esquelético y el ambiente (O’Regan \& Nöe, 2001).

\section{Discriminación, métrica intrínseca y procedimientos psicofísicos}

Aunque el tipo de procedimiento y análisis empleado en este escrito guarda más similitud con estudios psicofísicos sobre estimación de longitudes (Ono, 1967; Schiffman, 1965) que con procedimientos del AEC, un aspecto importante es que la estimación está en función de una acción y no en función de una escala en abstracto, como ocurre en los estudios psicofísicos. Bajo esta perspectiva se argumenta que la estimación de una dimensión del estímulo a partir de un significado conductual, y mediante una métrica intrínseca, permite ampliar las descripciones conductuales para los estudios en percepción y en psicofísica (Tonneau, 2011; Zuriff, 1972), es decir, la descripción aparentemente subjetiva de la sensación de un sujeto frente a una es- 
timulación física y que tradicionalmente el conductismo radical rechaza (Savage, 1966), puede revisitarse desde una aproximación en la que la estimación que el individuo hace de una magnitud del estímulo es objetiva (y no subjetiva como se ha asumido) dado que hace referencia a los posibilitadores de conducta (o significado conductual) que genera dicha estimulación.

Bajo estas consideraciones, los hallazgos de los dos experimentos presentados a la manera de las funciones psicofísicas (Figura 5) muestran que la percepción de la 'alcanzabilidad' de la línea horizontal a partir de la acción de algún miembro del propio sistema corporal con las que habitualmente se realiza esa clase de conductas (i.e., extremidades superiores) es más precisa y se hace con mayor seguridad, que cuando se utiliza un objeto ajeno al sistema corporal, como una barra ajustable, a pesar de que la altura de la barra coincida con la altura que el participante alcanzaría con sus extremidades superiores.

Esta diferencia en la ejecución coincide con el hecho de que se estiman con mayor facilidad las dimensiones de objetos que son familiares (como lo es el brazo para alcanzar las líneas), que las de objetos novedosos (Fillenbaum, Schiffman, \& Butcher, 1965; Schiffman, 1967). Este resultado sugiere que es posible que las estimaciones del alcance de la barra se vuelvan más precisas si se convierten en una situación usual para un individuo, pues existe evidencia experimental (Gibson \& Pick, 2000; O'Regan \& Nöe, 2001) que demuestra que con práctica las estimaciones perceptuales se vuelven más precisas.

Finalmente, la precisión en 'percibir si una conducta es posible', es relevante en términos adaptativos para el organismo, ya que permite anticipar el modo de acción particular dadas sus capacidades biomecánicas para enfrentar algún aspecto ambiental, como ejecutar un salto (Montagne, Cornus, Glize, Quaine, \& Laurent, 2000), cruzar sobre una hendidura (Burton \& Cyr, 2004; Jiang \& Mark, 1994), o simplemente colocar con la mano un objeto sobre una superficie (Rosenbaum, van Heugten, \& Caldwell, 1996). A partir de ello se puede identificar si el ambiente, o una superficie en particular, ofrece el soporte adecuado para que ocurra una conducta, lo que constituiría una restricción ambiental para el aprendizaje (Hinde \& Stevenson-Hinde, 1973; White, comunicación personal), pues así como el organismo debe poseer el suficiente soporte biológico y características propias de especie para desarrollar algún comportamiento, se asume que para lograr un aprendizaje (i.e., efecto de alguna contingencia de reforzamiento), el ambiente deberá constituir también un soporte conductual adecuado para el organismo implicado (Cabrera, Covarrubias, \& Jiménez, 2009; Johnston \& Turvey, 1980).

\section{Referencias}

Adolph, K. E. (1997). Learning in the development of infant locomotion. Monographs of the Society for Research in Child Development, 62, 1-163.

Adolph, K. E., Joh, A. S., \& Eppler, M. A. (2010). Infants' perception of affordances of 
slopes under high and low-friction conditions. Journal of Experimental Psychology: Human Perception and Performance, 36, 797-811.

Bertami, M., Yang, T. L. \& Proffitt, D.S. (1998). Relative size perception at a distance is best at eye level. Perception \& Psychophysics, 60, 673-682.

Blough, D. S. (1958). A method for obtaining psychophysical thresholds from the pigeons. Journal of the Experimental Analysis of Behavior, 1, 31-43.

Burton, G. \& Cyr, J. (2004). Gap crossing decisions in the sighted and visually impaired. Ecological Psychology, 16, 303-317.

Cabrera, F., Covarrubias, P., \& Jiménez, Á. A. (2009). Los sistemas conductuales desde una aproximación ecológica, En. J. Varela, F. Cabrera y J. J. Irigoyen (Eds.), Estudios sobre comportamiento y aplicaciones: Vol. 1, (pp. 31-50). México: Universidad de Guadalajara.

Cabrera, F., Sanabria, F., Jiménez, Á. A., \& Covarrubias, P. (2013). An affordance analysis of unconditioned lever pressing in rats and hamsters. Behavioural Processes, 92, 36-46.

Carello, C., Grosofsky, A., Reichel, F. D., Solomon, H. Y., \& Turvey, M. T. (1989). Visually perceiving what is reachable. Ecological Psychology, 1, 27-54.

Chang, C.-h., Wade, M. G., \& Stoffregen, T. A. (2009). Perceiving affordances for aperture passage in an environment-person-person system. Journal of Motor Behavior, 41, 495-500.

Costall, A. (2004). From Darwin to Watson (and cognitivism) and back again: The principle of animal-environment mutuality. Behavior and Philosophy, 32, 179-195.

Epstein, W. (1967). Perceptual learning resulting from exposure to a stimulus-invariant. The American Journal of Psychology, 80, 205-212.

Fillenbaum, S., Schiffman, R.H., \& Butcher, J. (1965). Perception of off-size versions of a familiar object under conditions of rich information. Journal of Experimental Psychology, 69, 298-303.

Gibson, J. J. (1966). The senses considered as perceptual systems. Boston, M.A.: Houghton Mifflin.

Gibson, J. J. (1979). The ecological approach to visual perception. New Jersey: Erlbaum.

Gibson, E. J., \& Pick, A. D. (2000). An ecological approach to perceptual learning and development. New York: Oxford University Press.

Heft, H. (1993). A methodological note on overestimates of reaching distance: Distinguishing between perceptual an analytical judgments. Ecological Psychology, 5, 255-271.

Heider, F. (1959). On perception, event structure and psychological environment. Psychological Issues, 1, Monograph 3, 1-123.

Heyser, C. \& Chemero, A. (2012). Novel object exploration in mice: not all objects are created equal. Behavioural Processes, 89, 232-238.

Hinde, R. A. \& Stevenson-Hinde, J. (1973). Constraints on learning. Limitations and predispositions. London: Academic Press.

Honig, W. K. \& Urcuioli, P. J. (1981). The legacy of Guttman and Kalish (1956): 25 
years of research on stimulus generalization. Journal of the Experimental Analysis of Behavior, 36, 405-445.

Järvilheto, T. (1998). The theory of organism-environment system: I. Description of the theory. Integrative Physiological and Behavioral Science, 33, 317-330.

Jiang, Y. \& Mark, L. S. (1994). The effect of gap depth on the perception of whether a gap is crossable. Perception \& Psychophysics, 56, 691-700.

Johnston, T. D. \& Turvey, M. T. (1980). A sketch of an ecological metatheory for theories of learning. En. G.H. Bower (Ed.), The psychology of learning and motivation, Vol. 14 (pp. 147-205). New York: Academic Press.

Keller, F.S. y Schoenfeld, W. N. (1950). Principles of psychology. Copley Publishing Group: Massachusetts.

Konczak, J., Meeuwsen, H. J., \& Cress, M. E. (1992). Changing affordances in stair climbing: The perception of maximum climbability in young and older adults. Journal of Experimental Psychology: Human Perception and Performance, 18, 691-697.

Lombardo, T. J. (1987). The reciprocity of perceiver and environment. The evolution of James J. Gibson's ecological psychology. New Jersey: Lawrence Erlbaum Associates.

Mark, L. S., Nemeth, K., Gardner, D. Dainoff, M. J., Paasche, J., Duffy, M., \& Grandt, K. (1997). Postural dynamics and the preferred critical boundary for visually guided reaching. Journal of Experimental Psychology: Human Perception and Performance, 23, 1365-1379.

Marotta, J. J. \& Goodale, M. A. (2001). The role of familiar size in the control of grasping. Journal of Cognitive Neuroscience, 13, 8-17.

Martínez-Escudero, L., Gámiz, F., \& Gallo, M. (2012). El papel de la forma del objeto en la conducta exploratoria de ratas: relevancia de vértices y aristas. Trabajo libre

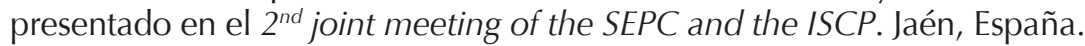

Montagne, G., Cornus, S., Glize, D., Quaine, F., \& Laurent, M. (2000). A perceptionaction coupling type of control in long jumping. Journal of Motor Behavior, 32, 37-43.

Nevin, J. N., Davison, M. C., \& Commons, M. L. (1991). Introduction. En M.L. Commons, J.A. Nevin, \& M.C. Davison (Eds.), Signal detection: Mechanisms, models, and applications. (pp. xvii-xxiv). New Jersey: Lawrence Erlbaum Associates, Publishers.

Ono, H. (1967). Difference threshold for stimulus length under simultaneous and nonsimultaneous viewing conditions. Perception and Psychophysics, 2, 201-207.

O'Regan, J., K., \& Nöe, A. (2001). A sensoriomotor account of vision and visual consciousness. Behavioral and Brian Sciences, 24, 939-1031.

Palmer, D. K. (2004). On the organism-environment distinction in psychology. Behavior and Philosophy, 32, 317-347.

Ríos-Checa, A., Quevedo, N., Ramírez, R., Gómez-Llanos, J., \& Jiménez Santa-Cruz, B. (2010). Sentarse sobre el dibujo de una silla. Revista Mexicana de Psicología, 27, 35-43.

Rochat, P. (1995). Perceived reachability for self and others by 3- to 5-year-old children and adults. Journal of Experimental Child Psychology, 59, 317-333. 
CABRERA ET AL.

Rosenbaum, D. A., van Heugten, C. M., \& Caldwell, G. E. (1996). From cognition to biomechanics and back: The end-state confort effect and the middle-is-faster effect. Acta Psychologica, 94, 59-85.

Savage, C.W. (1966). Introspectionist and behaviorist interpretations of ratio scales of perceptual magnitudes. Psychological Monographs: General and Applied, 80, 1-32.

Schiffman, H. R. (1967). Size-estimation of familiar objects under informative and reduced conditions of viewing. The American Journal of Psychology, 80, 229-235.

Schiffman, H. R. (1965). Size-estimationand the size of the measuring unit. Psychonomic Science, 3, 479-480.

Schoendeld, W. N., Antonitis, J. J., \& Bersh, P. J. (1950). Unconditioned response rate on the white rat in a bar-pressing apparatus. Journal of Comparative Physiological Psychology, 43, 41-48.

Schoenfeld, W. N., \& Cumming, W. W. (1963). Behavior and perception. En S Koch (Ed.), Psychology: A study of a science. Vol 5 (pp. 213-252). New York: McGrawHill.

Segal, E. (1959). The stability of operant level and its relations to deprivation. Journal of Comparative Physiological Psychology, 52, 713-716.

Skinner, B. F. (1938). The behavior of organisms. New York: Appleton Century Crofts.

Skinner, B. F. (1953). Science and human behavior. New York: The MacMillan Company.

Skinner, B. F. (1974). About Behaviorism. New York: Alfred A. Knopf, Inc.

Skinner, B.F. (1961). The design of cultures. Daedalus, 90, 534-546.

Staddon, J.E.R. (1983). Adaptive behavior and learning. London: Cambridge University Press.

Terrace, H.S. (1966). Stimulus control. En W.K. Honig (Ed.), Operant behavior. Areas of research and application (pp. 271-344). New York: Appleton Century Crofts.

Timberlake, W. (2004). Is the operant contingency enough for a science of purposive behavior? Behavior and Philosophy, 32, 197-229.

Tonneau, F. (2011). Magnitudes subjetivas y objetivas. Trabajo libre presentado en el

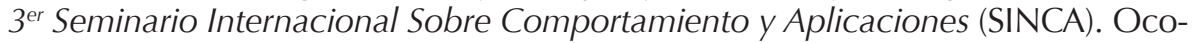
tlán, Jalisco, México

Tonneau, F. (2012). Holt's realism: New reasons for behavior analysis. En E.P. Charles (Ed.), A new look at new realism. The psychology of E. B. Holt. New Jersey: Transaction Publishers.

Tonneau, F., Kim Abreu, N., \& Cabrera, F. (2004). Sitting on the word "chair": Behavioral support, contextual cues, and the literal use of symbols. Learning and Motivation, 35, 262-273

Turvey, M. T. (1992). Affordances and prospective control: An outline of the ontology. Ecological Psychology, 4, 173-187.

von Hofsten, C. (2004). An action perspective on motor development. Trends in Cognitive Sciences, 8, 266-272.

von Hofsten, C., Vishton, P., Spelke, E. S., Feng, Q., \& Rosander, K. (1998). Predictive 
MÉTRICA INTRÍNSECA Y POSIBILITADORES DE ACCIÓN

action in infancy: tracking and reaching for moving objects. Cognition, 67, 255285.

Warren, W. H. (1984). Perceiving affordances: visual guidance of stair climbing. Journal of Experimental Psychology: Human Perception and Performance, 10, 683703.

Warren, W. H. (1995). Constructing an econiche. En J. Flach, P. Hancick, J. Caird, y K. Vicente. (Eds.), Global perspectives on the ecology of human-machine systems, Vol. 1. Erlbaum, Hillsdale: New Jersey.

Warren, W. H. (2006). The dynamics of perception and action. Psychological Review, 113, 358-389.

Warren, W. H. \& Whang, S. (1987). Visual guidance of walking through apertures: Body scaled information for affordances. Journal of Experimental Psychology: Human Perception and Performance, 13, 371-383.

Zuriff, G. E. (1972). A behavioral interpretation of psychophysical scaling. Behaviorism, 1, 118-133.

Recepción: Abril 15, 2013

Aceptación final: Agosto 19, 2013 


\section{CABRERA ET AL.}

86

\section{Apéndice A}

Resultados individuales de la proporción de respuestas como alcanzable en función de la altura del estímulo. Círculos grises representan Soporte Bajo y rombos vacíos representan Soporte Alto.

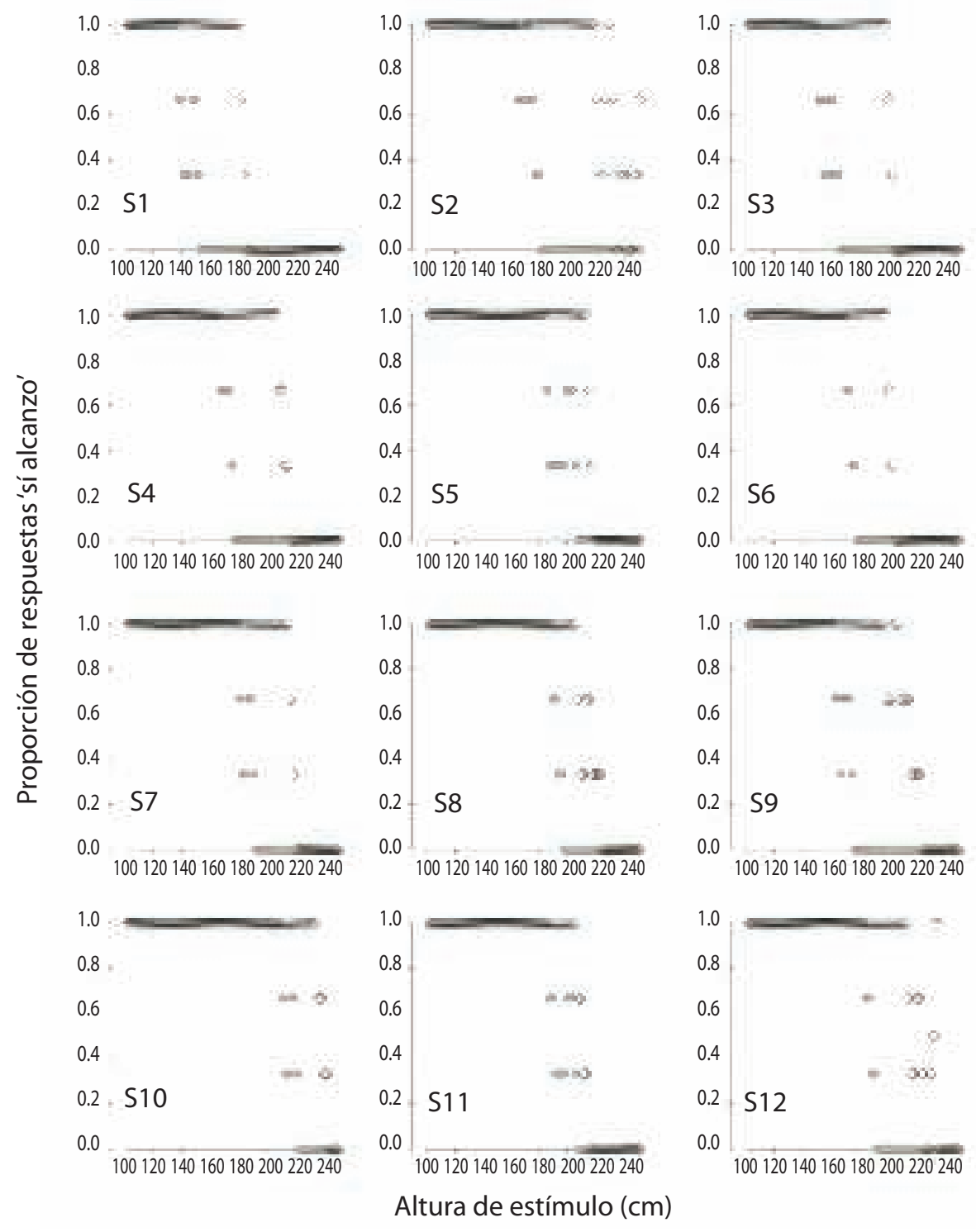




\section{Apéndice B}

Resultados individuales de la proporción de seguridad de las estimaciones en función de la altura del estímulo. Círculos grises representan Soporte Bajo y rombos vacíos representan Soporte Alto.
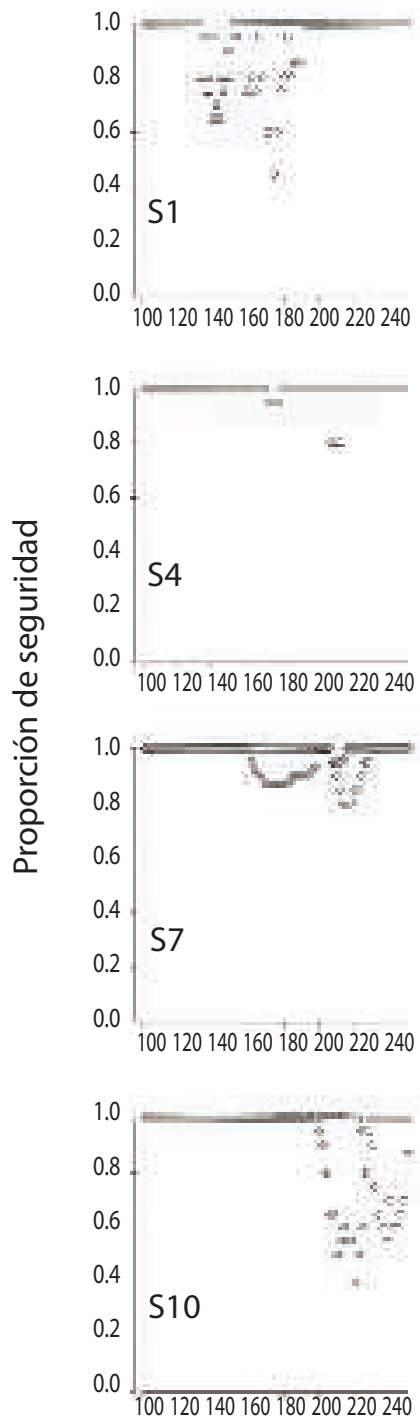
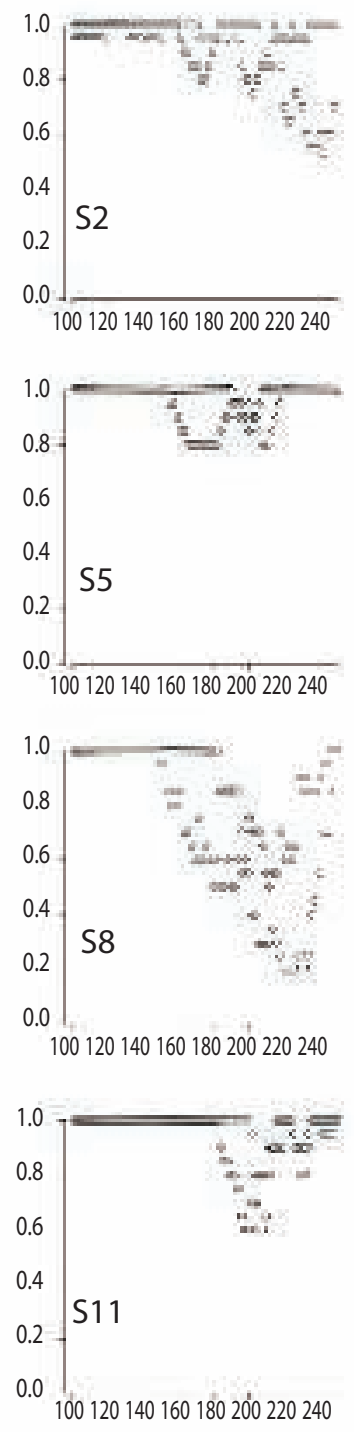

Altura de estímulo $(\mathrm{cm})$
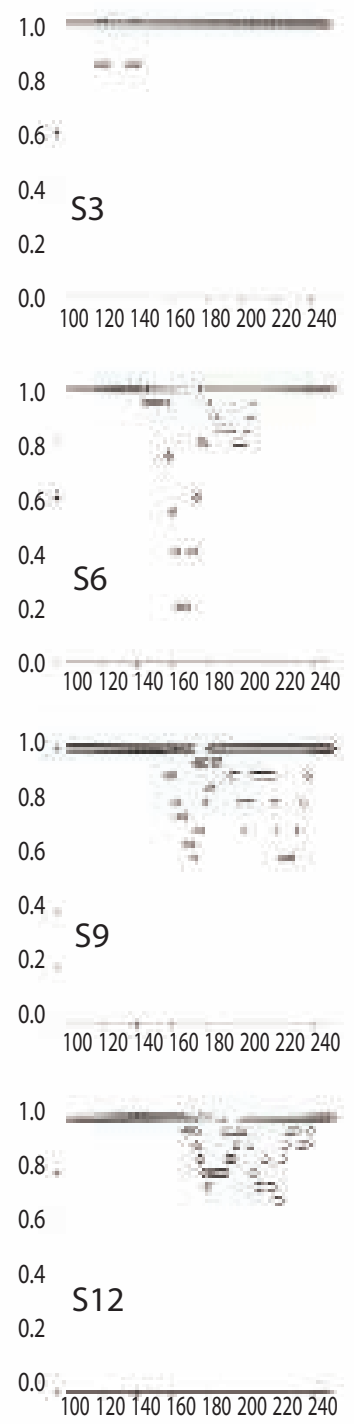

$0.0 \cdot \overline{100120140160180200220240}$ 
CABRERA ET AL.

88

\section{Apéndice C}

Resultados individuales de la proporción de respuestas como alcanzable en función de la métrica intrínseca del estímulo. Círculos grises representan Soporte Bajo y rombos vacíos representan Soporte Alto.

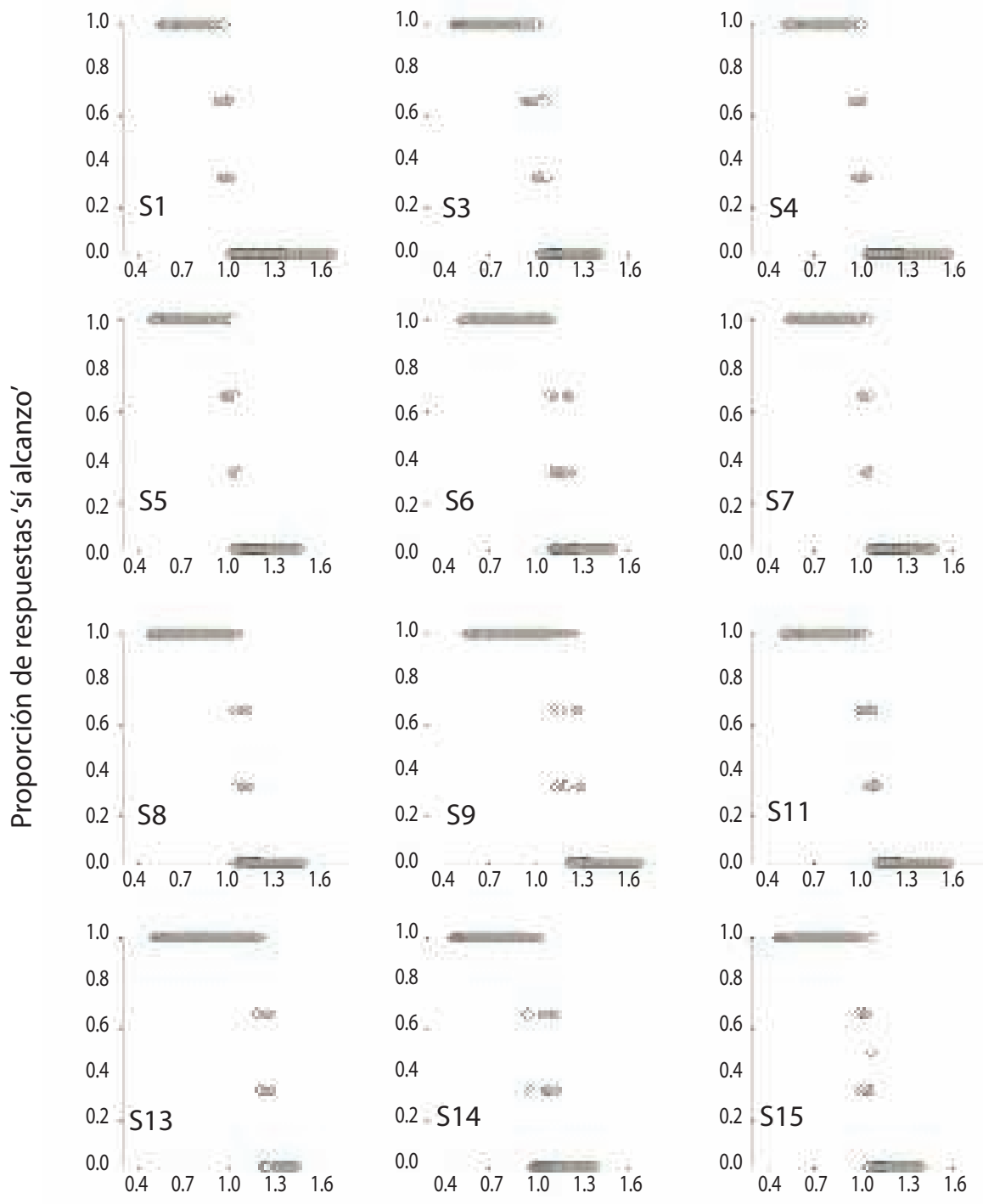

Altura de estímulo/alcance máx. de brazo 
MÉTRICA INTRÍNSECA Y POSIBILITADORES DE ACCIÓN

\section{Apéndice D}

Resultados individuales de la proporción de seguridad de las estimaciones como alcanzable en función de la métrica intrínseca del estímulo. Círculos grises representan Soporte Bajo y rombos vacíos representan Soporte Alto.
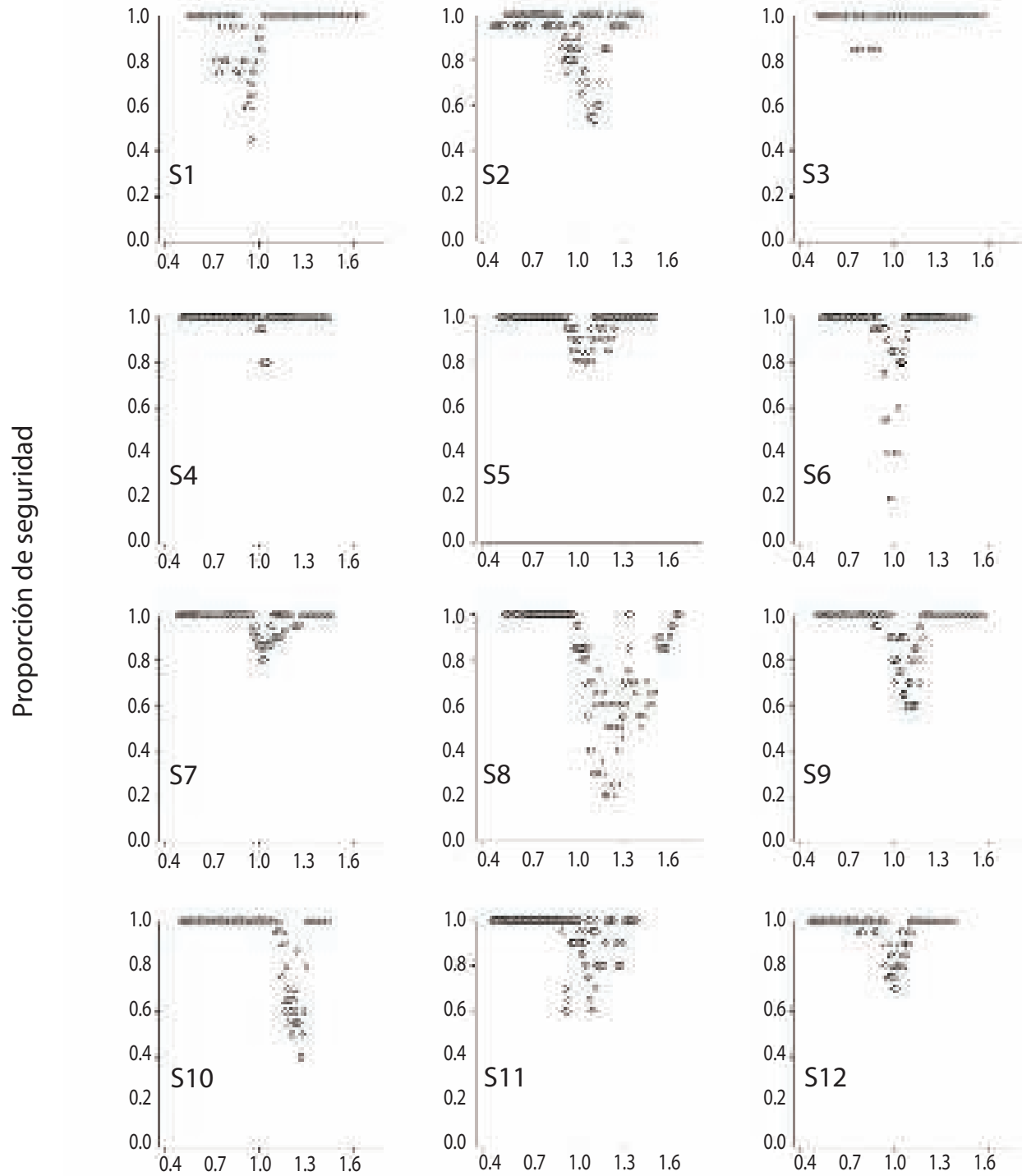

Altura de estímulo/alcance máx. de brazo 


\section{CABRERA ET AL.}

\section{Apéndice $\mathrm{E}$}

Resultados individuales de la proporción de respuestas como alcanzable en función de la métrica intrínseca del estímulo. Círculos grises representan Soporte Bajo y rombos vacíos representan Soporte Alto.

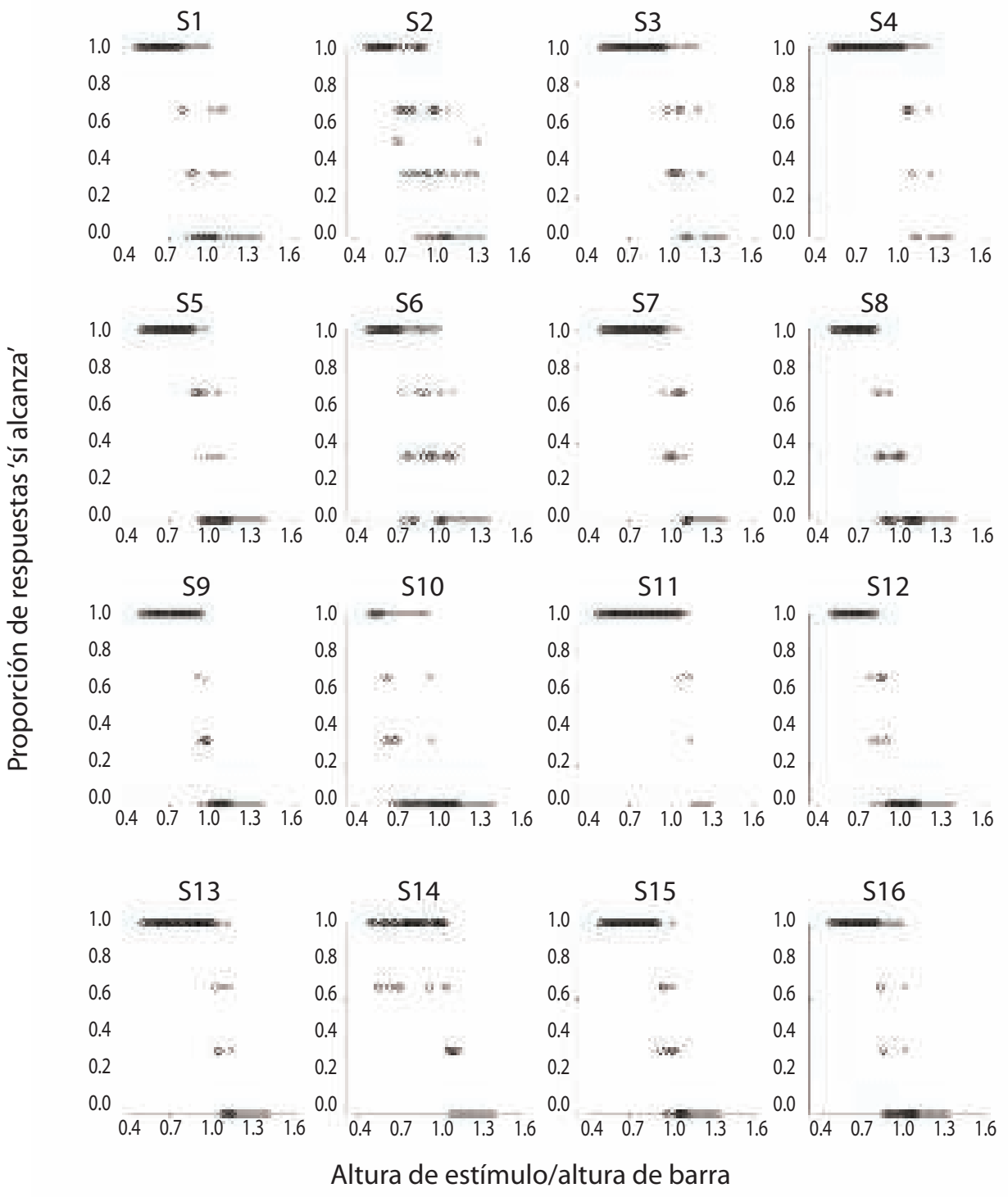


MÉTRICA INTRÍNSECA Y POSIBILITADORES DE ACCIÓN

\section{Apéndice $F$}

Resultados individuales de la proporción de seguridad de las estimaciones como alcanzable en función de la métrica intrínseca del estímulo. Círculos grises representan Soporte Bajo y rombos vacíos representan Soporte Alto.
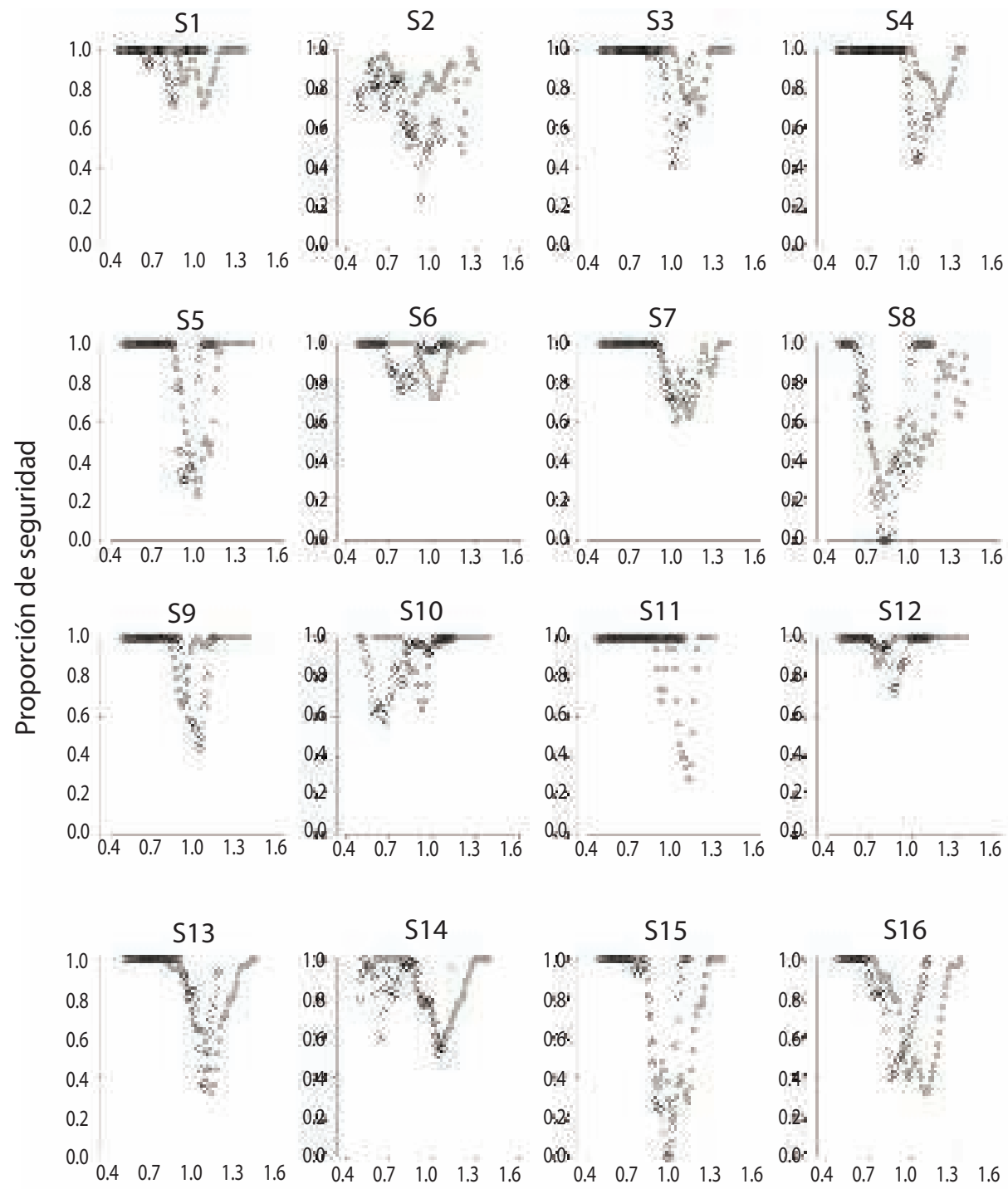

Altura de estímulo/altura de barra 